УДК 004.052.42

\title{
Towards automated error localization in C programs with loops*
}

\author{
Kondratyev D.A. (A.P. Ershov Institute of Informatics Systems SB RAS) \\ Promsky A.V. (A.P. Ershov Institute of Informatics Systems SB RAS) \\ The most recent trends in the C-light verification system are MetaVCG, semantic labels \\ appropriate for verification condition (VC) explanation and symbolic method of definite it- \\ erations. MetaVCG takes a C-light program together with some Hoare's logic and produces \\ on-the-fly a VC generator (VCG), which in turn processes the input program. Hoare's logic \\ for definite iterations is a good choice if we try to get rid of loop invariants. Finally, if \\ a theorem prover was unable to validate some VCs we could follow two ways. Obviously, \\ we could revise/enrich specifications or/and underlying proof theory to prove the truth \\ of VCs. Or, perhaps, we could concentrate upon establishment of falsity, which meant \\ there were errors in annotated program. This is where semantic labels play crucial role \\ providing some natural language comments about wrong VC as well as a back-trace to \\ the error location. The newly developed ACL2 heuristics to prove VC falsity is the main \\ theme of this paper.
}

Keywords: deductive verification, semantic label, error localization, C-light, automated theorem proof, C-lightVer, ACL2, MetaVCG, symbolic method of verification of definite iterations, proof strategy

\section{Introduction}

The C-light project [12] corresponds to the mainstream architecture of modern verification systems. It uses translation into an intermediate language (here, C-kernel) allowing to smooth over some hard corners of deductive verification. In order to improve efficiency we prefer domain specific verification condition (VC) generation, which means different generators for different program classes. Traditional approach implies manual reprogramming of VC generator (VCG). Instead, we adapted the MetaVCG approach of Moriconi and Schwarts [13]. For a given axiomatic system the MetaVCG automatically constructs an ordinary generator. The C-lightVer system is the implementation of the C-light project.

Are there any verification problems that cannot be solved by a two-level scheme or by 
MetaVCG? Indeed, there are. The loop invariants, for example. Another approach adapted in our project, so called symbolic verification of definite iterations [14], proposes solution for certain class of cycles over data structures. The loop execution is modeled symbolically by replacement function rep. Some results of such adaptation were discussed in [6, 8].

At the moment, the main theorem prover for C-light is ACL2 [5]. The classic induction in ACL2 is not powerful enough to handle VCs with replacement function rep, so the proof strategies are proposed in $[8,11]$.

The traditional deductive verification works ideally for a priori correct Hoare triple with true VCs. If some VCs are false the user must analyze the prover signals to understand what went wrong and to localize possible errors. An easy task for toy examples which becomes a real problem for real programs. Denney and Fisher developed the semantic labeling approach [3]. For every VC it provides the proof protocol (in axiomatic semantics) as well as localizing information up to the level of separate terms. A natural language explanation can be generated from such protocol. The MetaVCG approach allowed us to easily introduce semantic labels in our axiomatic systems $[6,7,12]$.

Now, after this background overview let us address the current problematic task, error localization for programs with loops. And again, situation is clear when ACL2 is able to discover truth or falsity. But often the answer is "unknown". Instead of trying to satisfy a VC we can use some strategies to check unsatisfiability. Since all variables in ACL2 are implicitly universally quantified, the existence quantifier appears in the negated VC. Thus, our previous strategies $[8,11]$ fail here and we need revised ones. Another requirement - the unsat strategies must work for loops with abrupt termination (i.e. in presence of break statement). Possible solution of this problem is discussed in this paper.

Related work. Some proof strategies deserve mention. For example, the system ACL2 (ml) [4] bases upon two methods. The first one is proof pattern recognition by means of statistical machine learning. The second one is symbolic searching for analogous lemma. However, the underlying theories may vary deeply, so machine learning is not very suitable for VC proof.

The SL-resolution is another well-known strategy [10]. Its inherent problem consists in necessary construction of useless resolvents. To oppose the growth of disjunct set the connection graph method was proposed [15]. In comparison to it, our strategy aims at the structure of literals, not disjuncts.

The goals of Constraint Logic Transformation project [2] remind ours. In the same time 
their strategies are suitable for predicate processing.

\section{Methods used in C-light project}

MetaVCG. The metagenerator takes proof rules and axioms as its input. Technically all of them represent patterns to be matched against $\mathrm{C}$ code [9]. The pattern language incorporates the first order logics and the $\mathrm{C}$ grammar. Expressions can contain nonterminal symbols, like uninterpreted predicate symbols or "fragment variables" [13] denoting code snippets. Affiliation of metadata with certain class in the pattern language is explicit [9]. For example, construction any_code $(S)$ can be matched against any sequence (including empty) of C statements, construction any_predicate $(P)$ can be matched against any predicate of specification language. Let vector $v=\left\langle v_{1} \ldots v_{k}\right\rangle$. Let each $\operatorname{expr}_{j}(1 \leq j \leq k)$ is result of replacement of all occurrences of term vector_element in the expression expr by $v_{j}$. Then construct vector_substitution $(T, v e c$, expr $)$ denotes the simultaneous replacement of all occurrences of each $v_{j}(1 \leq j \leq k)$ in the formula $T$ by expression $\operatorname{expr}_{j}$.

Semantic labeling. The idea of Denney and Fischer [3] consists in adding of semantic labels to the proof rules. Labels explain the result of rule application. We also use notation ${ }^{\lceil} t^{\rceil l}$, which means that the term $t$ is decorated with label $l$. Labels themselves take form $c(o, n)$, where $c$ is a concept (the term role), $o$ is a line range (in the source program) and $n$ is an auxiliary information.

In contrast to the original idea, the labels in our VCs form hierarchy more suitable to explanation generation [6]. We perform the depth search in the label tree and for each label its common text is expanded by corresponding pattern filled by line numbers. The text patterns for every label concept are similar to the $\mathrm{C}$ format strings, they are also fed to MetaVCG.

In order to support arbitrary label concepts a special construction (label t c) was added to the language of proof rules [7]. Here, $t$ is a term decorated by label and $c$ is a string (label type).

Symbolic method for the definite iterations. Consider a program fragment of the form for $\mathrm{x}$ in $\mathrm{S}$ do $\mathrm{v}:=\operatorname{body}(\mathrm{v}, \mathrm{x})$ end, where $S$ is a data structure, $x$ is a variable of type "element of $S$ ", $v$ is tuple of the loop variables excluding $x$, body represents the loop body which does not alter $x$ and terminates for every $x \in S$. The ways of modification of $S$ are quite restricted. The loop body can only contain assignments, the if statements (perhaps nested) 
ant the break statements. We call such for loops definite iterations. Let $v_{0}$ be the tuple of values of variables from $v$ at the loop entry point. Result of the whole definite iteration can be expressed by replacement operation $\operatorname{rep}(v, S, \operatorname{body}, n)$, where $\operatorname{rep}(v, S, \operatorname{body}, 0)=v_{0}$, $\operatorname{rep}(i, v, S, \operatorname{bod} y)=\operatorname{body}\left(\operatorname{rep}(i-1, v, S, \operatorname{bod} y), s_{i}\right)$ for all $i=1,2, \ldots, n$. If the break statement was executed during the $i$-th iteration of the body, the whole definite iteration continues, though $v$ does not change anymore: $\forall j(i \leq j \leq n) \operatorname{rep}(i, v, S, \operatorname{bod} y)=\operatorname{rep}(j, v, S, \operatorname{bod} y)$.

The MetaVCG allowed us to combine ideas of definite iterations and semantics labels in the form of the following pattern:

$\{P\} \operatorname{prog}\{$ (vector_substitution $(\mathrm{Q}, \mathrm{V}$, (label rep_iter $\operatorname{rep}(\mathrm{n}, \mathrm{v}, \mathrm{S}$, body).vector_element))\}

I- $\{$ any_predicate(P)\} any_code(prog)

for (int_var $(i)=0$; int_var $(i)<$ int_var(n); int_var $(i)++)$

admissible_construct(i, $\mathrm{n}, \mathrm{v}, \mathrm{S}$, body)

$\{$ any_predicate $(Q)\}$

where admissible_construct $(i, n, v, S$, body $)$ corresponds to an admissible body of definite iteration, int_var corresponds to an integer variable. The construct vector_substitution $(Q, v$, $\operatorname{rep}(n, v, S$, body).vector_element) denotes the simultaneous replacement of all occurrences of each $v_{t}(1 \leq t \leq$ length $(v))$ in the formula $Q$ by $\operatorname{rep}(n, v, S$, body $) . v_{t}$. The recursive definition of admissible construct is described in [8]. The algorithms of matching these patterns and program constructs have been implemented in the C-lightVer system [6-9]. The inference rule for downward iteration is defined similarly.

Automated generation of replacement operation. The replacement operation generation is based on translation [8] of loop body constructs into ACL2. Consider, for example, construction $b *$ :

$$
(b *(\ldots(\text { var expr }) \ldots) \text { result })
$$

Expression (var expr) denotes binding of variable var with the value of expression expr, which may depend on previously bound variables. The value of $b *$ is equal to value of result, which also can depend on bound variables. Values of variables from $v$ correspond to values of members of structure $f r$ of type frame. So, to model modification of some variable in $v$ we bind object $f r$ with new object which differs from the old one in the corresponding field. The abrupt termination is modeled by truth of boolean member loop-break of object $f r$. Instruction 
break is modeled by the following binding: ((when $t) f r$. Since condition when is true, such binding interrupts current block $b *$ and returns $f r$.

\section{Proof strategy for formulas with replacement operation}

The arguments of this strategy are implication $\psi$ containing expression $\operatorname{rep}(n, \ldots)$ and the premise $\phi$.

In the beginning we try to prove formula

$$
\phi \rightarrow \operatorname{rep}(n, \ldots) . \text { loop-break }
$$

by induction on $n$. If ACL2 succeeds, we add ( $\psi$-lemma-1) to the underlying theory. This lemma means that premise $\phi$ of implication $\psi$ represents situation when the loop is abruptly terminated. Then we try to prove $\psi$ using $(\psi$-lemma-1) and induction on $n$.

If ACL2 failed to prove ( $\psi$-lemma-1), we address to the formula

$$
\phi \rightarrow \neg r e p(n, \ldots) . \text { loop-break }
$$

by induction on $n$. Again, if ACL2 validates ( $\psi$-lemma-2) we add it to our theory in order to be used in the proof of $\psi$.

This strategy resembles one described in [8]. Indeed, both of them use the value of loop-break field. They are automatic. Finally, they are heuristics.

But differences also take place. First, this strategy is applied to any implication containing rep, whereas strategy from [8] analyses program postconditions only. Second, the latter one generates lemmas in the form of conjunction. The first conjunct is a VC and the second one establishes equality of antecedent from postcondition to the value of loop-break. Thus, lemmas generated in [8] have rather more complex structure.

\section{UNSAT strategy for VCs}

The argument $\omega$ of this strategy contains expression $\operatorname{rep}(n, \ldots)$ and has the following form:

$$
\forall x_{1} \ldots x_{n}\left(\phi_{1}\left(x_{1} \ldots x_{n}\right) \rightarrow \psi_{1}\left(x_{1} \ldots x_{n}\right)\right) \wedge \ldots \wedge\left(\phi_{m}\left(x_{1} \ldots x_{n}\right) \rightarrow \psi_{m}\left(x_{1} \ldots x_{n}\right)\right)
$$

In ACL2 all variables of $\omega$ are implicitly universally quantified. Note that every $\phi_{i}$ or $\psi_{i}$ is not forced to depend on all variables $x_{1} \ldots x_{n}$. But for simplicity we imply such dependence. 
Now let us prove $\neg \omega$ :

$$
\begin{aligned}
\left(\exists x_{1} \ldots x_{n}\left(\phi_{1}\left(x_{1} \ldots x_{n}\right) \wedge \neg \psi_{1}\left(x_{1} \ldots x_{n}\right)\right)\right) \vee \ldots & \\
& \left(\exists x_{1} \ldots x_{n}\left(\phi_{m}\left(x_{1} \ldots x_{n}\right) \wedge \neg \psi_{m}\left(x_{1} \ldots x_{n}\right)\right)\right)
\end{aligned}
$$

Let for each $i(1 \leq i \leq m) T_{i} \equiv \exists x_{1} \ldots x_{n}\left(\phi_{i}\left(x_{1} \ldots x_{n}\right) \wedge \neg \psi_{i}\left(x_{1} \ldots x_{n}\right)\right)$. To prove $\neg \omega$ it is sufficient to prove an arbitrary $T_{i}(1 \leq i \leq m)$. If we can find such $T_{i}$ the answer of strategy is $\mathrm{VC}$ is false. Otherwise, the answer is Unknown.

Now, to the proof of $T_{i}$. Obviously truth of formula

$$
T_{i}^{\prime} \equiv\left(\exists x_{1} \ldots x_{n} \phi_{i}\left(x_{1} \ldots x_{n}\right)\right) \wedge\left(\forall x_{1} \ldots x_{n}\left(\phi_{i}\left(x_{1} \ldots x_{n}\right) \rightarrow \neg \psi_{i}\left(x_{1} \ldots x_{n}\right)\right)\right)
$$

is sufficient to prove $T_{i}$. Let us denote subformula $\exists x_{1} \ldots x_{n} \phi_{i}\left(x_{1} \ldots x_{n}\right)$ as $U_{i}$ and the right subformula $\forall x_{1} \ldots x_{n}\left(\phi_{i}\left(x_{1} \ldots x_{n}\right) \rightarrow \neg \psi_{i}\left(x_{1} \ldots x_{n}\right)\right)$ as $V_{i}$. We need to prove both $U_{i}$ and $V_{i}$.

The process begins with interactive proof of $U_{i}$. Admittedly, sounds like undesirable step away from automated verification but the reasons are as follows. First, we use the weakest precondition calculus [8]. Therefore, $U_{i}$ cannot contain replacement operation. Only the specification functions that are known to user take place in $U_{i}$. Second, almost all automatic proof assistants have problems with existence quantifier. Third, our heuristics rests upon hypothesis of simple antecedents and complex consequents in implications that were generated by the wpcalculus. Based on this assumption, we try to automatize a more complex proof of $V_{i}$. If user cannot validate $U_{i}$ we suppose that the whole $T_{i}$ is false.

If user confirms $U_{i}$, the proof of $V_{i}$ starts. There are two possibilities. First, $V_{i}$ may be free of function rep. We simply pass this universally quantified formula to ACL2. Second, $V_{i}$ contains expression $\operatorname{rep}(n, \ldots)$. Anyway, since user does not know definition of rep, only the automated attempts are possible. The break statement can cause some trouble here because it complicates definition of rep. In this case, we address the strategy from Section 3.

\section{Example}

The goal of this experiment is to demonstrate execution of both strategies. This case study is also described in our on-line repository [1]. We deliberately made an error in the following function:

1. /*@ requires $(0<\mathrm{n})$ \&\& $(\mathrm{n}<=\operatorname{len}(\mathrm{a}))$;

2. ensures (grt-eql-cnt(n, key, a) $==0==>\backslash$ result $==0$ ) \&\& 
3.

$$
(\operatorname{grt}-\mathrm{eql}-\operatorname{cnt}(\mathrm{n}, \mathrm{key}, \mathrm{a})>0==>\backslash \text { result }==1)
$$

4. */

5. int grt_eql_key(int $\mathrm{n}$, int key, int $\mathrm{a}[])\{$

6. int $i$, result $=0$;

7. for $(i=0 ; i<n ; i++)\{$

8. if $(\mathrm{a}[\mathrm{i}]<\mathrm{key})\{$ result $=1$; break; $\}$

9. return result;\}

We suppose the reader is familiar with ACSL format of specifications. The logical function grt-eql-cnt counts the number of array elements greater or equal to key. Its definition is given in Appendix A. The program should look for array element that is greater than or equal to key and should return 1 or 0 according to the specification. Thus, the error is the use of operator < instead of operator $>=$ in if-condition of loop body. The result of intermediate translation into C-kernel looks like:

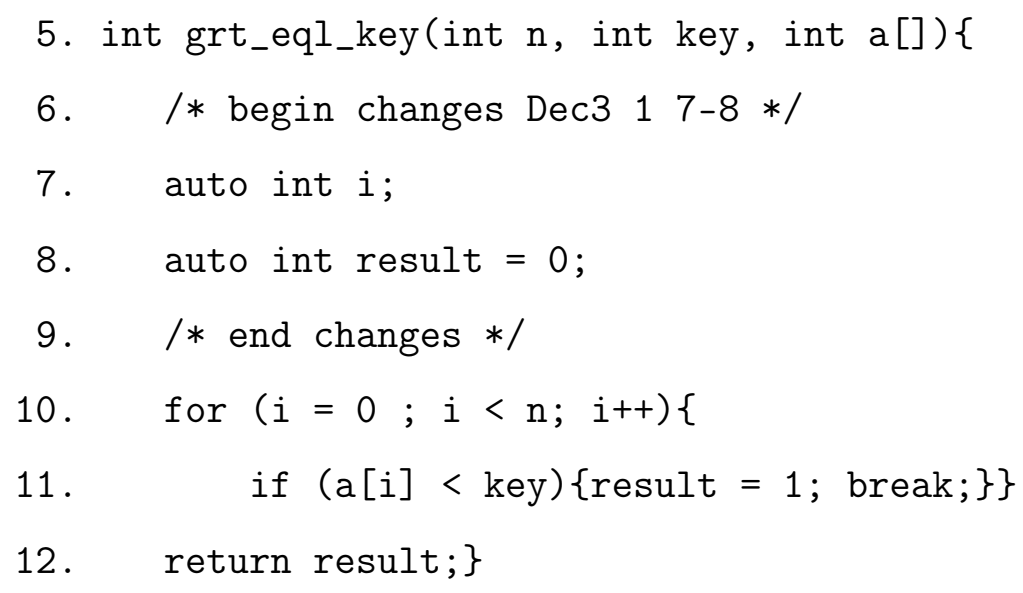

Note that neither specification nor definite iteration is modified by translator. The string "begin changes Dec3 1 7-8" stores data for the error localization protocol [12]. Of course, it did not appear because we knew about intentional error. It is inherent mechanism of our translation stage. In this particular case it declares that translation rule Dec3 for declarations [12] was used and strings 7-8 are its result. Due to the symbolic method VCG produces single $\mathrm{VC}(v c-1)$ : 


$$
\begin{aligned}
& \forall n, k e y, a \\
& \left(\left(\left\lceil_{0}<n \wedge n \leq \text { len }(a)^{\rceil^{\text {ass }} \_ \text {pre }(1)} \wedge n \in \operatorname{Int} \wedge \text { key } \in \text { Int } \wedge a \in \operatorname{Int} A r r \wedge\right.\right.\right. \\
& \left\lceil\operatorname{grt} \text {-eql-cnt }(n, k e y, a)=0^{\left\lceil a s s \_p o s t(2)\right.} \rightarrow\right.
\end{aligned}
$$

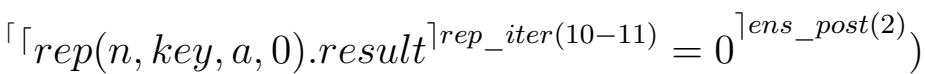

$$
\begin{aligned}
& \wedge \\
& \left({ }^{\lceil} 0<n \wedge n \leq \text { len }(a)^{\rceil a s s \_ \text {pre }(1)} \wedge n \in \operatorname{Int} \wedge \text { key } \in \text { Int } \wedge a \in \operatorname{Int} \text { Arr } \wedge\right. \\
& \left\lceil\operatorname{grt} \text {-eql-cnt }(n, k e y, a)>0^{\left\lceil a s s \_p o s t(3)\right.} \rightarrow\right. \\
& \left.{ }^{\ulcorner}{ } \operatorname{rep}(n, k e y, a, 0) \cdot r e s u l t{ }^{\rceil r e p \_i t e r(10-11)}=1^{\text {lens_post(3) }}\right)
\end{aligned}
$$

where IntArr is set of integral arrays. Function rep is defined in Appendix B. Semantic label ass_pre denotes hypothesis from precondition, ass_pre denotes hypothesis from postcondition, ens_post denotes goal from postcondition, rep_iter denotes substitution of replacement function [7]. As expected, $v c-1$ cannot be proved by SAT strategies, like those from [8, 11]. It is time to use our UNSAT strategy.

Formula $v c-1$ is a conjunction of two implications. Each of them uses function rep. The formula $U_{1}$ for the first conjunct $\phi$ looks like:

$$
\begin{gathered}
\exists n, k e y, a \\
0<n \wedge n \leq \text { len }(a) \wedge n \in \text { Int } \wedge \text { key } \in \text { Int } \wedge a \in \operatorname{Int} A r r \wedge \\
\text { grt-eql-cnt }(n, k e y, a)=0
\end{gathered}
$$

Since the user knows definition of grt-eql-cnt, he can prove $U_{1}$ in interactive mode. Therefore, formula $V_{1}$ for conjunct $\phi$ appears:

$$
\begin{aligned}
& \forall n, k e y, a
\end{aligned}
$$

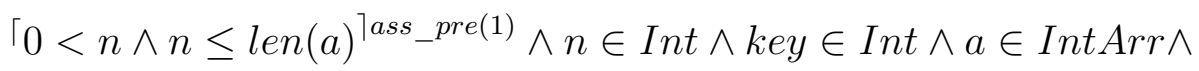

$$
\begin{aligned}
& \left\lceil\operatorname{grt} \text {-eql-cnt }(n, k e y, a)=0^{\left\lceil a s s \_p o s t(2)\right.} \rightarrow\right. \\
& { }^{\top} r_{r e p}(n, k e y, a, 0) . r e s u l{ }^{\dagger r e p \_i t e r(10-11)} \neq 0{ }^{\text {lens_post(2) }}
\end{aligned}
$$

This part of the proof is automatic. The presence of break in the loop body complicates things, so strategy from Section 3 enters on the scene. The corresponding $V_{1}$-lemma- 1 is as follows:

$$
\begin{gathered}
\forall n, k e y, a \\
0<n \wedge n \leq \text { len }(a) \wedge n \in \text { Int } \wedge \text { key } \in \text { Int } \wedge a \in \text { IntArr } \wedge \\
\text { grt-eql-cnt }(n, k e y, a)=0 \rightarrow \\
\text { rep }(n, \text { key }, a, 0) \text {.loop-break }
\end{gathered}
$$

This lemma deserves further explanation. Since $0<n$, the loop body is executed at least once. Amount of elements of $a$ greater or equal to key is also zero. Thus, all elements in sub-array 
$[0: n-1]$ are less than key. Control expression of the if statement contains wrong operator, so for such array the break statement is executed. $V_{1}$-lemma-1 was proved automatically in ACL2 by induction on $n$ and was added to the theory. Its lisp definition is given in Appendix C.

Finally, execution of break means that result $=1$. So, $V_{1}$-lemma-1 contributes in automatic proof of $V_{1}$. The lips definition of $V_{1}$ is given in Appendix D. As a result $v c-1$ is false and explanation for $V_{1}$ is produced. Let us consider this explanation.

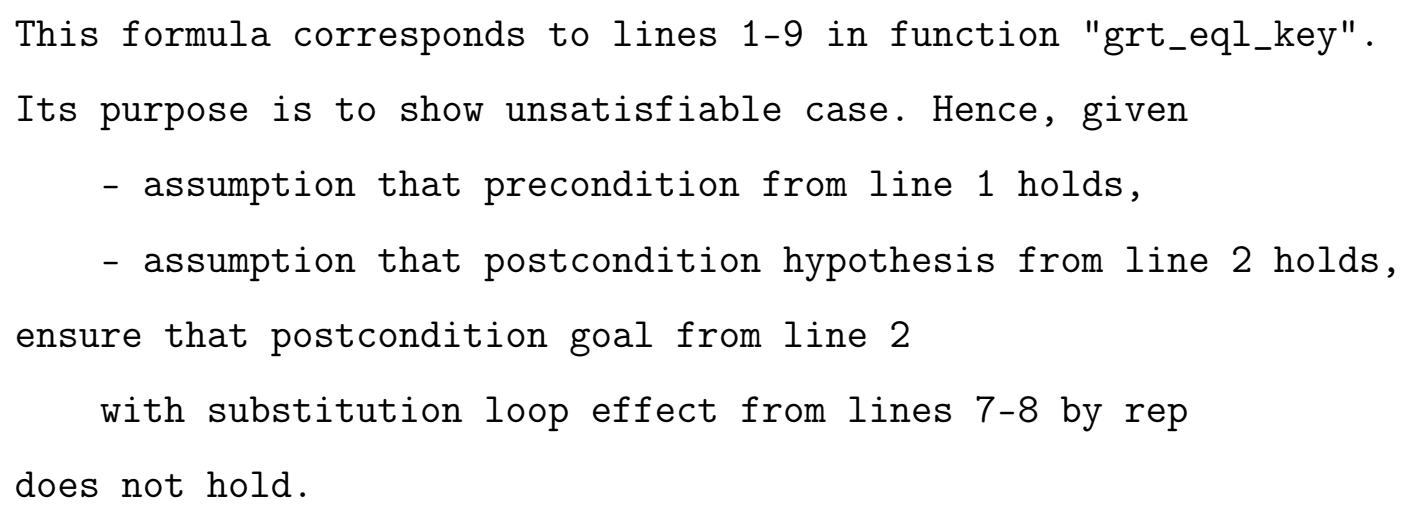

The error localization protocol [12] analyses semantic labels in $V_{1}$ and results of UNSAT strategy to generate the text above. Actually, labels in VCs contain string ranges of C-kernel program. This is where the commented information about translation rules proves to be useful. The location of possible error can be retranslated back to C-light program [12].

\section{Conclusion}

Many papers tend to demonstrate successful experiments avoiding the situation when verification fails. To fill this gap we discussed here some ideas about error localization in the C-light project. In case when the prover can confirm neither truth nor falsity, the traditional response if straightforward. User tries to reinforce the underlying theory in attempt to successfully reprove VCs. However, we do not discard bad scenario and simultaneously we try to check whether VCs are actually false. Another point of our current interest is verification of loops with break statement. We obtained some results in our experiments:

1. We devised the UNSAT strategy. It generates formula whose truth automatically implies falsity of original VC. At the moment such formula involves either interactive and automated proof.

2. We also propose the SAT strategy for VCs with operation rep. If abrupt termination of the loop is described in formula premises, a corresponding lemma will be added to the 
underlying theory.

These strategies are the new contribution relative to previous research $[6-8,11]$. Based on these methods, we conducted some experiments on error localization. We can also mention successful verification $[6,8]$ of function asum from the interface BLAS. The more complex data structures and the other functions from BLAS will be considered in future work.

\section{References}

1. Automated Error Localization in C Programs. URL: https://bitbucket.org/Kondratyev/verify-clight. Last accessed 30 Apr 2019.

2. De Angelis E., Fioravanti F., Pettorossi A., Proietti M.: Verification of Imperative Programs by Constraint Logic Program Transformation // Electronic Proceedings in Theoretical Computer Science. Vol. 129. P. 186-210.

3. Denney E., Fischer B. Explaining Verification Conditions // Lecture Notes in Computer Science. B.: Springer-Verlag, 2008. Vol. 5140. P. 145-159.

4. Heras J., Komendantskaya E., Johansson M., Maclean E. Proof-Pattern Recognition and Lemma Discovery in ACL2 // Lecture Notes in Computer Science. B.: Springer-Verlag, 2013. Vol. 8312. P. 389-406.

5. Hunt W. A., Kaufmann M., Moore J. S., Slobodova A. Industrial hardware and software verification with ACL2 // Philosophical Transactions of the Royal Society A: Mathematical, Physical and Engineering Sciences. 2017. Vol. 375. No. 2104. Article Number 20150399.

6. Kondratyev D. Implementing the Symbolic Method of Verification in the C-Light Project // Lecture Notes in Computer Science. Cham: Springer International Publishing AG, 2018. Vol. 10742. P. $227-240$.

7. Kondratyev D.A. The extension of the C-light project using symbolic verification method of definite iterations // XVII All-Russian Conf. of Young Scientists on Mathematical Modeling and Information Technology. Computational technologies. 2017. Vol. 22. P. 44-59. (In Russian)

8. Kondratyev D. A., Maryasov I. V., Nepomniaschy V. A. The Automation of C Program Verification by Symbolic Method of Loop Invariants Elimination // Modeling and Analysis of Information Systems. 2018. Vol. 25. No. 5. P. 491-505. (In Russian)

9. Kondratyev D. A., Promsky. A. V. Developing a self-applicable verification system. Theory and practice // Automatic Control and Computer Sciences. 2015. Vol. 49. No. 7. P. 445-452.

10. Kowalski R., Kuehner D. Linear Resolution with Selection Function // Artificial Intelligence. 1971. Vol. 2. No. 3-4. P. 227-260.

11. Maryasov I. V., Nepomniaschy V. A., Kondratyev D. A. Invariant Elimination of Definite Iterations over Arrays in C Programs Verification // Modeling and Analysis of Information Systems. 2017. Vol. 24. No. 6. P. 743-754.

12. Maryasov I. V., Nepomniaschy V. A., Promsky A. V., Kondratyev D. A. Automatic C Program 
Verification Based on Mixed Axiomatic Semantics // Automatic Control and Computer Sciences. 2014. Vol. 48. No. 7. P. 407-414.

13. Moriconi M., Schwarts R.L. Automatic Construction of Verification Condition Generators From Hoare Logics // Lecture Notes in Computer Science. B.: Springer-Verlag, 1981. Vol. 115. P. 363377.

14. Nepomniaschy V.A. Symbolic method of verification of definite iterations over altered data structures // Programming and Computer Software. 2005. Vol. 31. No. 1. P. 1-9.

15. Siekmann J., Wrightson G. An Open Research Problem: Strong Completeness of R. Kowalski's Connection Graph Proof Procedure // Lecture Notes in Computer Science. B.: Springer-Verlag, 2002. Vol. 2408. P. 231-252.

\section{A. The ACL2 definition of grt-eql-cnt}

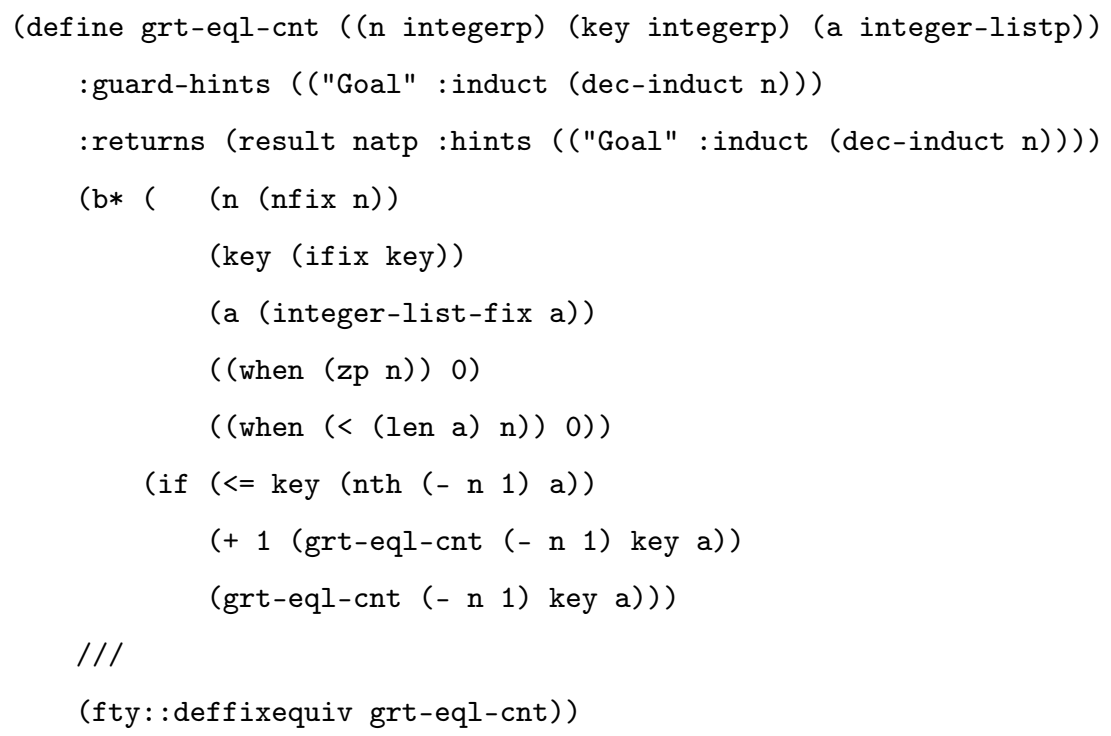

Due to recursive nature of grt-eql-cnt, induction is preferable when it comes to the proof of statements containing this function.

\section{B. The ACL2 definition of function rep}

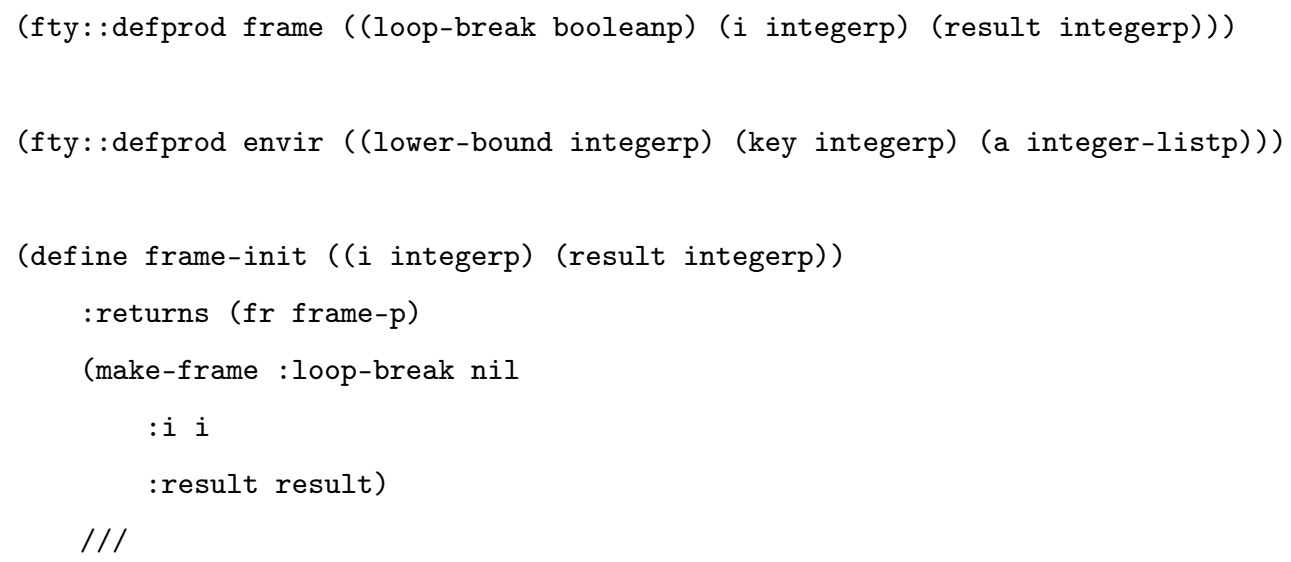


(fty: :deffixequiv frame-init))

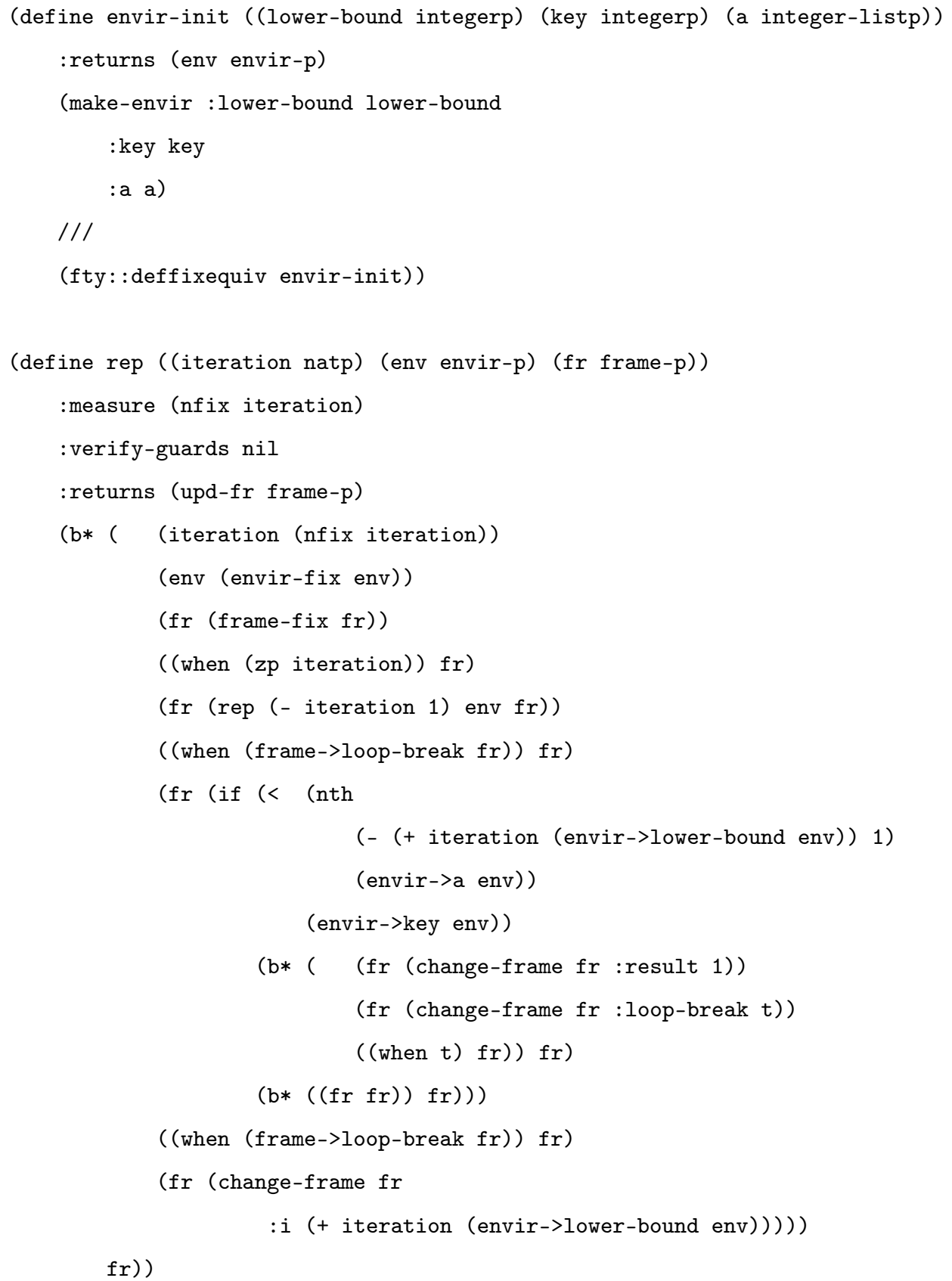

\section{The ACL2 definition of $V_{1}$-lemma-1}

(defrule v-1-lemma-1

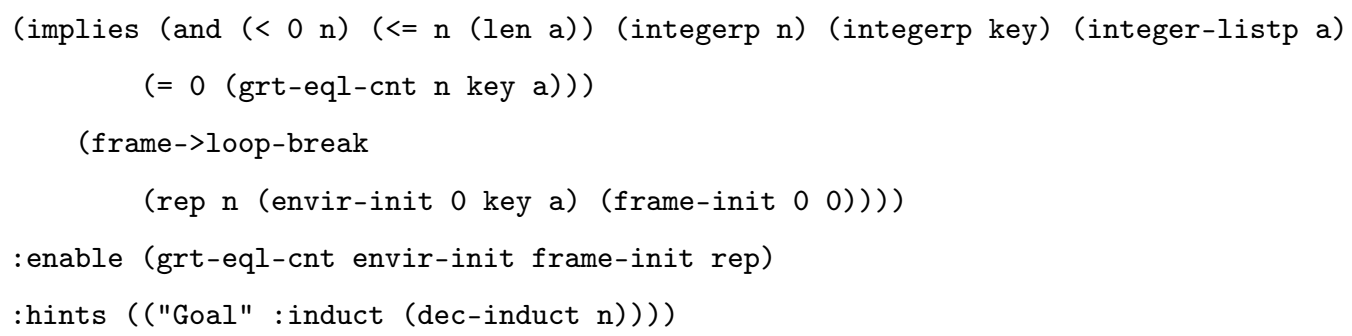

Construction of the form 
:hints (("Goal" :induct (dec-induct $\mathrm{n}))$ )

prompts ACL2 to use induction on $n$.

\section{The ACL2 definition of lemma $V_{1}$}

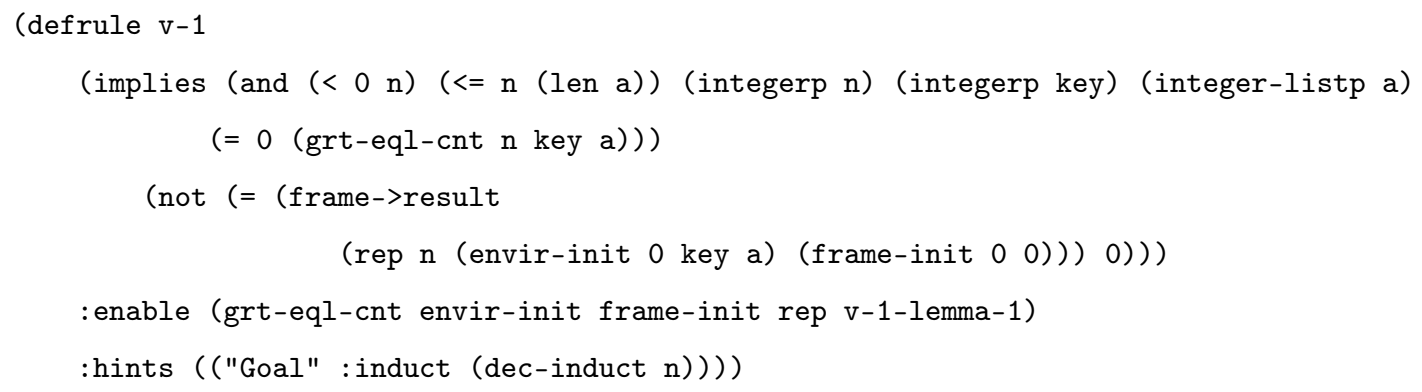


